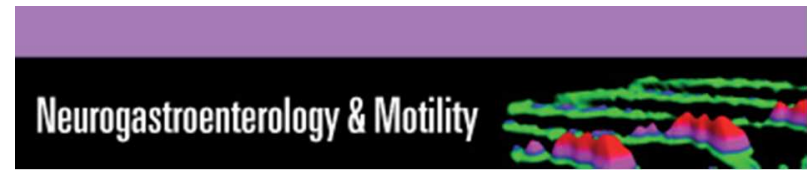

\title{
Cine MRI assessment of motility in the unprepared small bowel in the fasting and fed state: Beyond the breath-hold
}

\begin{tabular}{|c|c|}
\hline Journal: & Neurogastroenterology and Motility \\
\hline Manuscript ID & NMO-00087-2018.R2 \\
\hline Manuscript Type: & Original Article \\
\hline Date Submitted by the Author: & 06-Aug-2018 \\
\hline Complete List of Authors: & $\begin{array}{l}\text { Khalaf, Asseel; University of Nottingham School of Medicine, Nottingham } \\
\text { Digestive Diseases Centre; Nottingham University Hospitals NHS Trust, } \\
\text { NIHR Nottingham Biomedical Research Centre (BRC) } \\
\text { Nowak, Adam; Nottingham University Hospitals NHS Trust, NIHR } \\
\text { Nottingham Biomedical Research Centre (BRC) } \\
\text { Menys, Alex; University College London Medical School, Centre for Medical } \\
\text { Imaging } \\
\text { Marciani, Luca; University of Nottingham School of Medicine, Nottingham } \\
\text { Digestive Diseases Centre; Nottingham University Hospitals NHS Trust, } \\
\text { NIHR Nottingham Biomedical Research Centre (BRC) } \\
\text { Taylor, Stuart; University College London Medical School, Centre for } \\
\text { Medical Imaging } \\
\text { Spiller, Robin; University of Nottingham School of Medicine, Nottingham } \\
\text { Digestive Diseases Centre; Nottingham University Hospitals NHS Trust, } \\
\text { NIHR Nottingham Biomedical Research Centre (BRC) } \\
\text { Gowland, Penny; University of Nottingham School of Physics and } \\
\text { Astronomy, Sir Peter Mansfield Imaging Centre } \\
\text { Moran, Gordon; University of Nottingham School of Medicine, Nottingham } \\
\text { Digestive Diseases Centre; Nottingham University Hospitals NHS Trust, } \\
\text { NIHR Nottingham Biomedical Research Centre (BRC) } \\
\text { Hoad, Caroline; Nottingham University Hospitals NHS Trust, NIHR } \\
\text { Nottingham Biomedical Research Centre (BRC); University of Nottingham } \\
\text { School of Physics and Astronomy, Sir Peter Mansfield Imaging Centre }\end{array}$ \\
\hline Key Words: & Gastrointestinal motility, MRI, Cine MRI, Fasting, Fed state \\
\hline
\end{tabular}


Khalaf 1

Cine MRI assessment of motility in the unprepared small bowel in the fasting and fed state: Beyond the breath-hold

Running head: Optimised analysis technique for the assessment of small bowel motility

Asseel Khalaf ${ }^{1,2}$, Adam Nowak ${ }^{2}$, Alex Menys ${ }^{3}$, Luca Marciani ${ }^{1,2}$, Stuart A. Taylor ${ }^{3}$, Robin C. Spiller ${ }^{1,2}$, Penny A. Gowland ${ }^{4}$, Gordon W. Moran ${ }^{1,2}$, and Caroline L. Hoad ${ }^{2,4}$

${ }^{1}$ Nottingham Digestive Diseases Centre, School of Medicine, University of Nottingham UK

${ }^{2}$ NIHR Nottingham Biomedical Research Centre (BRC), Nottingham University Hospitals NHS Trust and the University of Nottingham, Nottingham, UK.

${ }^{3}$ Centre for Medical Imaging, Division of Medicine, UCL, London, UK

${ }^{4}$ Sir Peter Mansfield Imaging Centre, School of Physics and Astronomy, University of Nottingham, Nottingham UK

\section{Corresponding author:}

Dr Caroline Hoad

Senior Research Fellow

Sir Peter Mansfield Imaging Centre, School of Physics and Astronomy, University of Nottingham

NIHR Nottingham Biomedical Research Centre (BRC), Nottingham University Hospitals NHS Trust and the University of Nottingham, Nottingham, UK

Nottingham NG7 2UH, U.K.

E-mail: caroline.I.hoad@nottingham.ac.uk 
Khalaf 2

\section{Abstract}

Background:

The symptoms of functional bowel disorders are common in postprandial but investigations are generally undertaken in the fasted state using invasive procedures. MRI provides a non-invasive tool to study the gastro-intestinal tract in an unperturbed, fed state. The aim of this study was to develop a technique to assess small bowel motility from cine MRI data in the unprepared bowel in fasting and fed states.

Methods:

Fifteen healthy volunteers underwent a baseline MRI scan after which they consumed a $400 \mathrm{~g}$ soup. Subjects then underwent a postprandial scan followed by further scans at regular intervals. Small bowel motility was assessed using singleslice bTFE cine MRI.

An optimised processing technique was used to generate motility data based on power spectrum analysis of voxel-signal changes with time. Inter-observer variability $(n=15)$ and intra-observer $(n=6)$ variability were assessed. Changes in the motility index were compared between fasted and immediate postprandial state.

Results:

Excellent agreement between observers was seen across the range of motility measurements acquired, with intraclass correlation coefficient (ICC) of 0.979 $(p<0.0001)$ and Bland-Altman limits of agreement $95 \% \mathrm{Cl}:-28.9$ to $45.9 a . u$. Intraobserver variability was low with ICC of 0.992 and 0.960 (2 observers, $P<0.0001$ ).

Changes from the fasted to immediately postprandial state showed an average increase of $122.4 \pm 98.7 \%(n=15)$ 


\section{Khalaf 3}

Conclusion:

This optimised technique showed excellent inter and intra observer agreement. It was sensitive to changes in motility induced feeding. This technique will be useful to study contractile activity and regional patterns along the gastrointestinal tract under physiological conditions.

KEY WORDS: Gastrointestinal motility; MRI; cine MRI; fasting; fed state 
Khalaf 4

\section{Key points:}

- Many patients' symptoms are more prevalent after eating a meal. However most investigative MR paradigms are undertaken in the fasting patient using bowel preparation, which does not represent normal physiological motion.

- This study developed an optimised technique for the analysis of gut motility from cine MRI images, specifically addressing the differences in appearance and motility of the small bowel wall in unprepared cine MR images in both fasting and fed states.

- With the clinical availability of MRI scanners, this technique has the potential to improve our knowledge of the pathophysiology of gastrointestinal tract under normal physiological conditions. 


\section{Khalaf 5}

\section{Introduction}

Conventional manometry of small bowel motility has provided valuable insights into motor function pathophysiology of the gastrointestinal tract ${ }^{1,2}$. The technique has limitations, with naso-duodenal or oro-duodenal intubation being a difficult, uncomfortable and invasive procedure for patients. Moreover, manometry techniques are not generally used in the lower sections of the small bowel due to difficulties with access and the invasiveness of the technique ${ }^{3,4}$. Furthermore, misinterpretation of the manometry recordings can occur if non-occlusive contractions occur and large spacing between ports mean that motor patterns can be mis-defined ${ }^{5}$. The tube may also interfere with normal feeding making it particularly difficult to study physiological changes from the fasting state and the effect of nutrient intake.

Over the last 10 years, MRI has proven to be a useful tool to probe the unprepared physiology of the gastro-intestinal tract ${ }^{6-9}$. It is particularly suitable for longitudinal or repeated studies, and its versatility allows for multiple physiological parameters to be monitored in a single scanning session. Magnetic resonance enterography (MRE) is used to evaluate the small bowel after the ingestion of an oral contrast agent. It involves distending the bowel artificially to produce detailed images of the bowel wall 10 and induces bowel wall motion to move the large amount of oral contrast agent through the GI tract, which can then be studied using cine MRI.

Motility measurements following oral contrast preparation using a cine MRI acquisition have made significant advances in recent years ${ }^{8,11-17}$, but to date quantification of wall motion has either involved looking for contractions across the lumen ${ }^{13,14,16}$ or using registration methods ${ }^{15,17}$, which work well in the deliberately distended bowel where the walls are clearly visualised. However, bowel distension with a hypo-osmotic solution may not be truly physiological and so cannot study true 
Khalaf 6

fasting motility patterns and may not represent the full range of motility patterns in the postprandial state. The ability to study motility in the postprandial state, or the transition between the two states, has many potential advantages in furthering our understanding of physiology and the origin of symptoms which many patients experience after feeding. Moreover, it is particularly important for the pharmaceutical sciences because the rate and extent of drug dissolution and absorption from solid oral dosage forms is highly dependent upon gastrointestinal motility ${ }^{18,19}$. Furthermore, the use of bowel distension limits the use of MRE in paediatric and elderly populations.

The unprepared small bowel can be imaged using the same high spatial and temporal resolution cine acquisitions as for the prepared bowel ${ }^{11,12,14}$. However, the post processing techniques used to parameterise the motility may need to be refined for images, which do not delineate the small bowel wall clearly and show different patterns of motility.

The aim of this study was to develop an analysis technique to assess motility from cine MRI data acquired in the fasted and fed, unprepared small bowel. Inter- and intra-observer variability, and the sensitivity to changes in motility caused by feeding were investigated.

\section{Materials and methods}

This study was approved by the local Ethics Committee of the University of Nottingham (H19062014). This study is registered on www.clinicaltrials.gov with identifier NCT02717117. All subjects gave informed written consent. The study design, subjects, and data sets used have been reported previously ${ }^{20}$. 
Khalaf 7

\section{Unprepared bowel data acquisition}

Fifteen healthy volunteers (age $29 \pm 10$ years, BMI $24 \pm 5 \mathrm{~kg} / \mathrm{m}^{2}$ ) were recruited from the local campus population. Subjects with any disease or taking medication (e.g. loperamide, codeine, metoclopramide, hyoscine butylbromide, mebeverine, ondansetron) that affects gastric emptying or small bowel transit were excluded. Standard MRI exclusion criteria were applied.

This study was open label. Subjects were scanned using a 1.5T Philips Achieva MRI scanner (Philips Healthcare, Best, the Netherlands) using the 16 element torso (XLTORSO, Philips Healthcare) coil at the Sir Peter Mansfield Imaging Centre, University of Nottingham. They underwent a baseline fasting scan defined as $t=-20$ min time point. They were asked to consume a soup meal (cream of chicken soup $(400 \mathrm{~g})$ (or mushroom for vegetarians) (Heinz, Wigan, UK)) ${ }^{21}$ within 20 min then the subjects underwent a first immediate postprandial scan (defined as $t=0 \mathrm{~min}$ ). MRI data collection was subsequently repeated every 15 minutes for the first 60 minutes where the subjects remained in the scanner and then every 30 minutes up to 270 minutes where subjects were allowed to leave the scanner between scans if they chose $\mathrm{t}^{20}$. The subjects were scanned using a range of sequences. At each time point scans were acquired to assess small bowel motility ${ }^{8}$ using a single slice bTFE cine MRI acquisition (with reconstructed in-plane resolution $1.49 \times 1.7 \mathrm{~mm}^{2}$, slice thickness $10 \mathrm{~mm}$, echo time $(T E)=1.52 \mathrm{~ms}$, repetition time $(T R)=3.0 \mathrm{~ms}$, flip angle $80^{\circ}$, SENSE 2.0), of 1 minute free-breathing, temporal resolution of $1 \mathrm{~s}$, this was repeated at six contiguous parallel coronal planes through the small bowel as previously described ${ }^{20}$. The total scan time for motility was 6 min. The subjects were instructed to take shallow gentle breaths for the duration of the motility acquisition. 
Khalaf 8

\section{Data analysis}

\section{Motility assessment}

Free breathing data were processed using GIQuant ${ }^{\mathrm{TM}}$ (Motilent, Ford, UK). The algorithm corrects respiratory motion ${ }^{22}$ before applying the non-linear optic flow registration as described previously ${ }^{15}$ to correct local deformation caused by bowel wall motion and model intensity changes caused by luminal flow. The data output from the image registration were further analysed using a customised graphical user interface written in MATLAB ${ }^{\circledR}$ (MathWorks, Natick, MA, USA).

On MRI the unprepared small bowel has a very different appearance to the prepared bowel required for MRE (Figure 1). In the prepared bowel there is clear definition of the bowel walls and obvious peristaltic motion is visible through the time series across most of the small bowel. In the unprepared bowel, the bowel wall is not always visible and bolus movement of the chyme between segments is common post-prandially. Therefore a different approach for quantifying the motility of the unprepared small bowel was developed, based on the registration parameter $C^{15}$ which represents the change in signal intensity between time points, within a defined region of interest $(\mathrm{ROI})$ placed over the small bowel loops. This parameter is modelled simultaneously with the deformation during the registration process and is intended to capture any signal intensity changes not occurring from in-plane motion (i.e. through plane motion and flow $)^{15}$.

To allow sensitivity to both oscillatory events such as mixing of contents during peristalsis and forward propulsion of boluses of chyme, the power spectrum analysis of the image registration parameter $\mathrm{C}$ was developed similar to that proposed by van der Paardt et $\mathrm{al}^{23}$ and Sprengers et $\mathrm{al}^{24}$. Initially, to remove zero frequency data, the 
Khalaf 9

mean of $\mathrm{C}$ through time for each pixel was calculated and subtracted from each pixel value (Figure 2A). Then for each pixel in the data, the power spectrum (Fourier transform of time course of mean-subtracted $C$, smoothed to reduce noise and with the first time point removed to eliminate data not in the steady state, and then multiplied by its complex conjugate) was calculated (Figure 2B), generating data with frequency information up to $0.48 \mathrm{~Hz}$. The area under the power spectrum was calculated as a summary metric $\left(A \cup C_{\text {power spectrum }}\right)$, and maps created to visualise the regions of higher motility. This metric was intended to reflect both segmental oscillations and bolus movement of contents, typically seen post-prandially ${ }^{25}$. When regions of interest were defined, average data for the ROI was calculated from the pixel by pixel measurements within the ROI of the $A U C_{\text {power spectrum maps. }}$

\section{Observer variability}

To determine the variability in the results due to observer definition of the region of the small bowel loops, the following analyses were carried out.

1. Inter-observer variability: Two observers, 1 experienced ( $\mathrm{CH}$ over 10 years) in viewing small bowel MRI data and 1 inexperienced (AK less than 2 years) drew regions around all the visible small bowel segments across the 6 coronal slices acquired. This was repeated for all 15 subjects scanned at all time points pre and post-test meal. The number of regions depended on the spatial separation of the different bowel loops. If there was large amount of visceral fat separating the loops, more regions were drawn to encompass all the small bowel loops.

2. Intra-observer variability: The same two observers drew regions around the 
Khalaf 10

visible small bowel segments from only six subjects (chosen to have different body composition: 3 normal BMI (22.3, $\left.22.922 .9 \mathrm{~kg} / \mathrm{m}^{2}\right)$ and 3 high BMI (30.6, $27.1,29.1 \mathrm{~kg} / \mathrm{m}^{2}$ ). The changing body composition resulted in images with very different contrast of the edges of the small bowel loops and therefore represented the maximum range of tissue contrast that would be seen across all subjects. Regions were defined by drawing the ROls to encompass only the visible small bowel loops ignoring intra-abdominal visceral fat and other tissues; the regions were defined twice on the images with at least one month interval between observations.

\section{Changing motility in response to feeding using two motility metrics}

We examined the strength of correlation between two motility analysis techniques using the total power ( $\mathrm{AUC}_{\text {power spectrum }}$ ) and the standard deviation of the Jacobian $\left(\mathrm{SD}_{\mathrm{JAC}}\right.$, a previous published metric for motility $\left.{ }^{8}\right) . \mathrm{SD}_{\mathrm{JAC}}$ looks at the geometric changes from image registration and is currently used for small bowel motility in the prepared bowel. In addition, we showed how both metrics change with feeding by looking at the mean change in the metric between the fasted $(t=-20 \mathrm{~min})$ and the immediately postprandial data $(\mathrm{t}=0 \mathrm{~min})$.

\section{Statistical analyses}

All statistical analysis was carried out using Graph Pad Prism 7.0 (La Jolla, USA). All data were tested for normality using the D'Agostino and Pearson normality test. Inter-observer variability was investigated by using a Bland-Altman plot to determine the $95 \%$ confidence limits of agreement. Correlation between observers was 
Khalaf 11

measured using the Intra-class correlation coefficient (ICC) using a 2-way random effects model, with a single rater and absolute agreement ${ }^{26}$. Intra-observer variability was also investigated with Intra-class correlation coefficients using a 2-way mixed effects model and single rater and absolute agreement. The $95 \%$ confidence limits of agreement were also calculated. Pearson correlation coefficient was used to measure the strength of correlation between the measurement of motility using $A \cup C_{\text {power spectrum }}$ and $\mathrm{SD}_{\mathrm{JAC}}$ for the fasting and immediately postprandial data sets.

\section{Results}

Visualisation of high motility regions

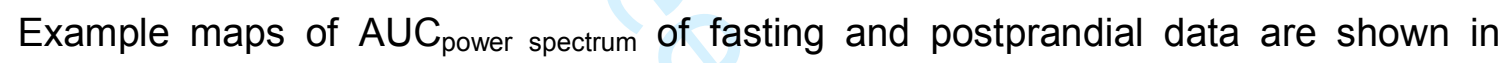
Figure 3 for one subject. Example maps of SDJAC are also shown for comparison.

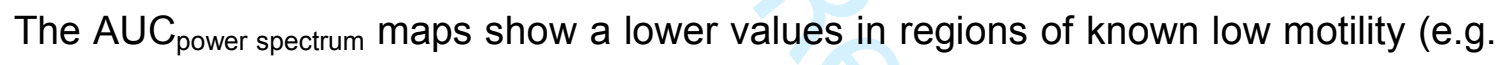
liver) compared to the $S D_{J A C}$ maps. There also appears to be a larger change

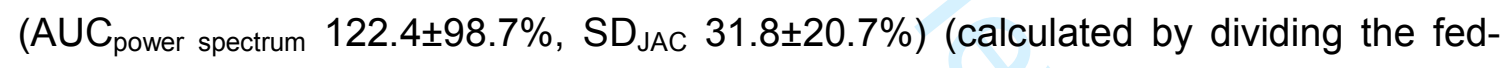
fasted data by the fasted data across all the 15 subjects) across the small bowel between the fasted and immediately postprandial states which suggests the $A U C_{\text {power }}$ spectrum maps may have better range to define the differences between sporadic movements occurring during fasting and large scale movements following ingestion of the meal.

\section{Inter-observer variability of small bowel motility}

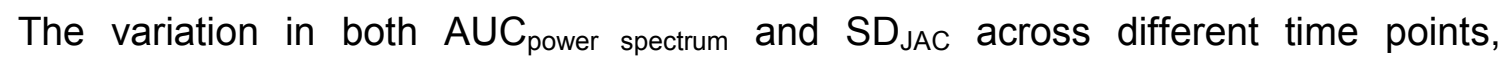
averaged over all healthy volunteers at each time point, and covering all regions of 
Khalaf 12

small bowel from the 6 slices, measured by each observer is shown in figure $4 \mathrm{~A}$ (error bars shown are SEM). This graph shows low measured motility at baseline in the fasting state followed by a significant increase post-prandially which then persists for the majority of the imaging period. The degree of correlation between two observers was assessed using the ICC to be 0.979 and $p<0.0001, n=195$ (Figure 4B). Inter-observer variability was assessed using the Bland Altman plot ${ }^{27}$ (Figure 4C) which showed a mean difference of 8.5 a.u. between small bowel motility measurements, with a $95 \%$ confidence interval of -28.9 to 45.9 a.u. as indicated by the upper and lower dotted lines (figure 4C).

Intra-observer variability of small bowel motility

The correlation between the two analyses for $\mathrm{AUC}_{\text {power spectrum }}$ performed by each observer was also assessed using the ICC and Bland-Altman limits of agreement (Table 1), showing good agreement between the analyses (ICC > 0.9 for all data).

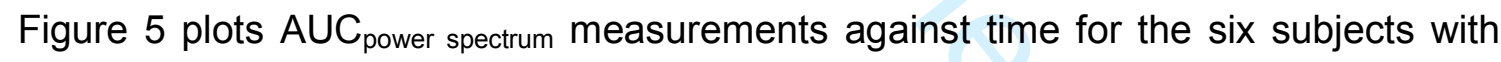
normal and high BMI, showing good agreement between analyses at most time points for both body compositions.

\section{Changing motility in response to feeding}

The subjects showed an increase between their fasting and initial postprandial

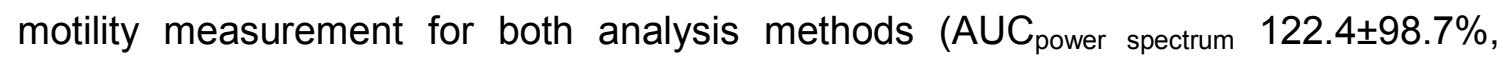

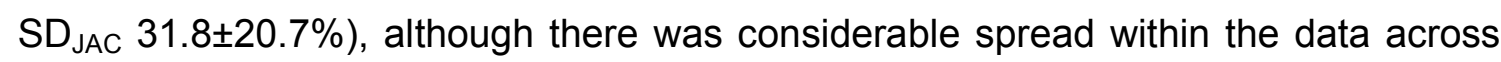
the subjects (Figure 6). The correlation between the techniques was significant $(r=0.9, p<0.0001, n=30)$. 
Khalaf 13

\section{Discussion}

This study has described and evaluated an optimised technique for the analysis of gut motility from MRI images, specifically addressing the differences in appearance and motility of the small bowel wall in unprepared bowel MR images, compared to images obtained following bowel preparation with oral luminal contrast agent. The images in fasting and fed conditions showed poor small bowel wall definition in places. Coupled with the different motility patterns of bolus propulsion as well as peristalsis meant previously published analysis techniques, which are based on geometric changes over the measurement period to generate the motility metric, were not as appropriate to use in fasting and fed studies. Maps of $A \cup C_{\text {power spectrum }}$ indicated a better discrimination of the bowel tissue compared to the more widely published $\mathrm{SD}_{\mathrm{JAC}}{ }^{8,15,28}$ with lower noise in the regions where non/low-motility is expected, and a bigger range of motility indices. The proposed method utilises changes in signal intensities that occur when the small bowel contents move between segments in regions showing bolus movement of contents (duodenum and jejunum) as well as those exhibiting more oscillatory motion (ileum), rather than looking for continuous motion throughout the time series.

The appearance of the unprepared bowel, which may contain multiple collapsed loops which are not always easily identified, means the definition of the ROls is more subjective than for the prepared bowel with distended lumen. However our interobserver variability data suggested that ROI definition had a small effect on the results compared to the changes in motility seen following eating the meal. Excellent correlation between observers was seen across the whole range of $A \cup C_{\text {power spectrum }}$ measurement acquired and the Bland-Altman limits of agreement were low compared 
Khalaf 14

to the range of values measured. The postprandial changes over time show a rapid increase in motility following ingestion of the soup meal with levels returning towards baseline much later after the meal had been consumed ${ }^{20}$. Intra-observer variability was also low with a small range of Bland-Altman limits of agreements for both observers across two very different body shapes. The ICC was excellent with all data greater than 0.9 .

This study presents an optimised analysis of MRI data to assess the motility of the unprepared fasting and fed small bowel. The MRI method proposed removes the need for intubating subjects, which can be a stressful procedure, often requiring fluoroscopy to place the catheter and rarely covering the entire length of the small bowel. Orocecal transit times can be measured using breath tests ${ }^{29}$ but these include changes due to gastric empting and do not give information about the specific motor function of the small bowel but are an indirect measure of small bowel motility. Scintigraphy transit studies ${ }^{30}$ involving ionising radiation do not provide information about the motor patterns seen in the small bowel. The proposed techniques (including further frequency analysis of the power spectra) will be useful to study the time scales of contractile activity and regional patterns along the gastrointestinal tract in health and disease. Information on small bowel motility can be obtained in conjunction with mapping the bowel liquid pockets using MRI ${ }^{31}$ furthering understanding of the effects of motility on the fluid environment of the bowel. These combined insights could help with advancing in vitro/in vivo predictive dissolution studies of oral dosage forms under similar, undisturbed conditions.

The data from the normal and high BMI subjects would indicate that there is a potential for over estimating motility in the higher BMI subjects. These subjects all presented with high motility indices postprandially, however only one of the 3 showed 
Khalaf 15

high baseline data. These larger motility indices may have been measured as regions of high signal intensity in the fat as it moves into and out of the imaging plane during respiratory motion. As this may not be fully corrected by the registration algorithm these movements will be interpreted as bowel motility. Further studies of higher BMI subjects is needed to understand the factors contributing to the larger motility metric measured and whether poor registration is a factor.

Other factors, which could also influence the signal intensity, are field inhomogeneities and metallic artefacts. To some extent, overall changes in image intensity across the image due to these factors are removed from the $A \cup C_{\text {power spectrum }}$ analysis by using the registration parameter $\mathrm{C}$ which models the signal changes and not the absolute values. An empty bowel has a different intensity to a filled bowel, however movement of the contents either between loops or from one section to another show similar changes in intensity levels. Other meal contents should have similar motility patterns to the meal in this study, but would need investigating to determine whether the sensitivity is the same as the soup meal, particularly for a more solid meal, which may have a much lower signal intensity in the small bowel.

There were limitations to our study. Due to the time consuming nature of drawing all the individual ROls on the motility data the intra-observer repeated measurements were confined to just 6 subjects, not the full 15 available (used for the inter-observer data). Drawing of ROls for a single time point took around 5-10 minutes depending on the anatomy including the loading of each data set into the software. However, the intra-observer data were chosen from subjects who had very different small bowel anatomical appearances due to their differing BMls, providing the observers with contrasting data for drawing the regions. Smoothing of the data before calculating the power spectrum reduces the effects of isolated poor mis-registration of the data. 
Khalaf 16

However it will not eliminate the effects completely and these datasets will slightly overestimate the small bowel motility present.

In conclusion, this study describes an optimised analysis technique to evaluate small bowel motility in the physiological fasting and postprandial states using registered cine MRI datasets. This method showed excellent agreement between measurements of intra and inter observers as well as showing the sensitivity of the technique to changes in motility induced by ingestion of a meal. Cine MRI scanning is available on most clinical scanners worldwide therefore future studies have real potential to translate and improve our knowledge of the small bowel environment in health and disease.

\section{Acknowledgements}

This study was supported by the National Institute for Health Research Nottingham Biomedical Research Centre. The views expressed are those of the authors and not necessarily those of the National Health Service (NHS, U.K.), the National Institute for Health Research (NIHR, U.K.) or the Department of Health. This study was funded by a scholarship from the Kuwait University and from a research grant from the Nottingham University Hospitals' Charity (PP-Gordon Moran-Nov14). The views expressed are those of the author(s) and not necessarily those of the NHS, the NIHR or the Department of Health.

LM is supported in part by Award \# HHSF223201510157C: "In Vivo Predictive Dissolution (IPD) to Advance Oral Product Bioequivalence (BE) Regulation" by the U.S. Food and Drug Administration (FDA); this manuscript represents the position of the authors and not necessarily that of the FDA.

AM is the CEO of Motilent Limited, a medical imaging analysis company. 
Competing Interests: the remaining authors have no competing interests.

AK, CLH, LM and GWM designed the research. AK, AN recruited the patients. AK collected the data. AK, AM, and CLH, analysed the data. AK, CLH, LM and GWM wrote the manuscript draft. All authors revised the final manuscript.

\section{References}

1. Hansen MB. Small intestinal manometry. Physiol Res. 2002;51(6):541-556.

2. Malagelada JR, Stanghellini V. Manometric Evaluation of Functional Upper Gut Symptoms. Gastroenterology. 1985;88(5):1223-1231.

3. Malagelada C, Malagelada JR. Small Bowel Motility. Curr Gastroenterol Rep. Jun 2017;19(6):26.

4. Seidl H, Gundling F, Pfeiffer A, Pehl C, Schepp W, Schmidt T. Comparison of small-bowel motility of the human jejunum and ileum. Neurogastroenterol Motil. Aug 2012;24(8):e373-380.

5. Arkwright JW, Dickson A, Maunder SA, et al. The effect of luminal content and rate of occlusion on the interpretation of colonic manometry. Neurogastroenterol Motil. Jan 2013;25(1):E52-E59.

6. Schwizer W, Steingoetter A, Fox M. Magnetic resonance imaging for the assessment of gastrointestinal function. Scand $J$ Gastroenterol. Nov 2006;41(11):1245-1260.

7. Fruehauf H, Menne D, Kwiatek MA, et al. Inter-observer reproducibility and analysis of gastric volume measurements and gastric emptying assessed with magnetic resonance imaging. Neurogastroenterol Motil. Sep 2011;23(9):854861.

8. Menys A, Taylor SA, Emmanuel A, et al. Global Small Bowel Motility: Assessment with Dynamic MR Imaging. Radiology. Nov 2013;269(2):442-449.

9. Marciani L, Garsed KC, Hoad CL, et al. Stimulation of colonic motility by oral PEG electrolyte bowel preparation assessed by MRI: comparison of split vs single dose. Neurogastroenterol Motil. Oct 2014;26(10):1426-1436.

10. Griffin N, Grant LA, Anderson S, Irving P, Sanderson J. Small bowel MR enterography: problem solving in Crohn's disease. Insights Imaging. Jun 2012;3(3):251-263.

11. Wakamiya M, Furukawa A, Kanasaki S, Murata K. Assessment of Small Bowel Motility Function With Cine-MRI Using Balanced Steady-State Free Precession Sequence. J Magn Reson Imaging. May 2011;33(5):1235-1240.

12. Ohkubo H, Kessoku T, Fuyuki A, et al. Assessment of Small Bowel Motility in Patients With Chronic Intestinal Pseudo-Obstruction Using Cine-MRI. Am J Gastroenterol. Jul 2013;108(7):1130-1139. 
13. Menys A, Atkinson D, Odille F, et al. Quantified terminal ileal motility during MR enterography as a potential biomarker of Crohn's disease activity: a preliminary study. Eur Radiol. Nov 2012;22(11):2494-2501.

14. Froehlich JM, Waldherr C, Stoupis C, Erturk SM, Patak MA. MR motility imaging in Crohn's disease improves lesion detection compared with standard MR imaging. Eur Radiol. Aug 2010;20(8):1945-1951.

15. Odille F, Menys A, Ahmed A, Punwani S, Taylor SA, Atkinson D. Quantitative assessment of small bowel motility by nonrigid registration of dynamic MR images. Magn Reson Med. Sep 2012;68(3):783-793.

16. Bickelhaupt S, Froehlich JM, Cattin R, et al. Differentiation between active and chronic Crohn's disease using MRI small-bowel motility examinations - Initial experience. Clin Radiol. Dec 2013;68(12):1247-1253.

17. Hahnemann ML, Nensa F, Kinner S, Gerken G, Lauenstein TC. Motility mapping as evaluation tool for bowel motility: initial results on the development of an automated color-coding algorithm in cine MRI. J Magn Reson Imaging. Feb 2015;41(2):354-360.

18. Oberle RL, Amidon GL. The influence of variable gastric emptying and intestinal transit rates on the plasma level curve of cimetidine: an explanation for the double peak phenomenon. $J$ Pharmacokinet Biopharm. Oct 1987;15(5):529-544.

19. Oberle RL, Chen TS, Lloyd $\mathrm{C}$, et al. The influence of the interdigestive migrating myoelectric complex on the gastric emptyign of liquids. Gastroenterology. Nov 1990;99(5):1275-1282.

20. Khalaf A, Hoad CL, Menys A, et al. MRI assessment of the postprandial gastrointestinal motility and peptide response in healthy humans. Neurogastroenterol Motil. Aug 312017.

21. Moran GW, Leslie FC, McLaughlin JT. Crohn's disease affecting the small bowel is associated with reduced appetite and elevated levels of circulating gut peptides. Clin Nutr. Jun 2013;32(3):404-411.

22. Hamy V, Dikaios N, Punwani S, et al. Respiratory motion correction in dynamic MRI using robust data decomposition registration - Application to DCE-MRI. Med Image Anal. Feb 2014;18(2):301-313.

23. van der Paardt MP, Sprengers AMJ, Zijta FM, Lamerichs R, Nederveen AJ, Stoker J. Noninvasive Automated Motion Assessment of Intestinal Motility by Continuously Tagged MR Imaging. J Magn Reson Imaging. Jan 2014;39(1):916.

24. Sprengers AMJ, van der Paardt MP, Zijta FM, et al. Use of continuously MR tagged imaging for automated motion assessment in the abdomen: A feasibility study. J Magn Reson Imaging. Aug 2012;36(2):492-497.

25. Silverthorn DU. Human Physiology: an integrated Approach. London: Pearson; 2012.

26. Koo TK, Li MY. A Guideline of Selecting and Reporting Intraclass Correlation Coefficients for Reliability Research. J Chiropr Med. Jun 2016;15(2):155-163.

27. Bland JM, Altman DG. Statistical methods for assessing agreement between two methods of clinical measurement. Lancet. Feb 08 1986;1(8476):307-310. 


\section{Khalaf 19}

28. Plumb AA, Menys A, Russo E, et al. Magnetic resonance imaging-quantified small bowel motility is a sensitive marker of response to medical therapy in Crohn's disease. Aliment Pharmacol Ther. Aug 2015;42(3):343-355.

29. Geypens B, Bennink R, Peeters M, et al. Validation of the lactose-[C-13]ureide breath test for determination of orocecal transit time by scintigraphy. $J \mathrm{Nucl}$ Med. Sep 1999;40(9):1451-1455.

30. Maurer AH. Gastrointestinal Motility, Part 2: Small-Bowel and Colon Transit. J Nucl Med Technol. Mar 2016;44(1):12-18.

31. Mudie DM, Murray K, Hoad CL, et al. Quantification of gastrointestinal liquid volumes and distribution following a $240 \mathrm{~mL}$ dose of water in the fasted state. Mol Pharm. Sep 2014;11(9):3039-3047. 
Khalaf 20

\section{Tables}

Table 1

\begin{tabular}{|c|c|c|}
\hline & ICC & Bland-Altman \\
\hline Observer 1 & $\begin{array}{c}0.992 \\
P<0.0001 n=78\end{array}$ & $\begin{array}{l}\text { MD 6.1 a.u. } \\
95 \% \mathrm{Cl} \\
-7.2 \text { to } 19.4 \text { a.u. } \\
\text { Measurement Range }(27.2-254.5 \text { a.u. })\end{array}$ \\
\hline Observer 2 & $\begin{array}{c}0.960 \\
P<0.0001 n=78\end{array}$ & $\begin{array}{c}\text { MD } 1.0 \text { a.u. } \\
95 \% \mathrm{Cl} \\
-41.2 \text { to } 43.19 \text { a.u. } \\
\text { Measurement Range (28.4 - } 281.0 \text { a.u. })\end{array}$ \\
\hline
\end{tabular}

Table legends

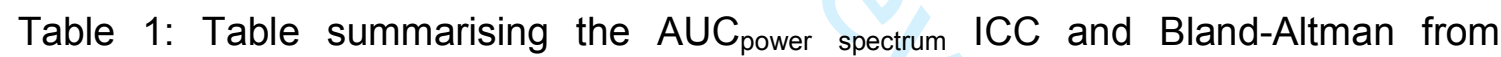
observer 1 and observer 2. MD - Mean Difference; $\mathrm{Cl}$ - Confidence Interval

\section{Figure legends}

Figure 1. Figure illustrating the difference between $(A)$ the prepared bowel with clear definition of the bowel wall and bright luminal contents (data not from the current study, bowel preparation of $2 \%$ mannitol with $0.2 \%$ locust bean gum) and (B) the unprepared bowel with less visible bowel wall and brighter contents only when chyme moves into the segment.

Figure 2 A. Graph showing the variation in intensity of the C parameter with time for 3 abdominal regions. Solid black line is a small ROI from the upper small bowel 
Khalaf 21

(jejunum), dashed black line is a small ROI from the lower small bowel (ileum), solid grey line is a small ROI from the ascending colon representing a known low motility region of the GI tract. B. Corresponding power spectrum of the data in A.

Figure 3. Example of motility maps generated by the software for a single volunteer across the 6 slices acquired, visualising the areas of high motility. A and B illustrate the fasting and the fed state motility maps. $\mathrm{C}$ represents the different motility maps generated by the $A U C_{\text {power spectrum }}$ and $\mathrm{SD}_{\mathrm{JAC}}$ motility parameters. S: slice number. Regions of small bowel have been highlighted on the images.

Figure 4. A. Graph showing the mean small bowel motility assessed with $A \cup C_{\text {power }}$ spectrum and $\mathrm{SD}_{\mathrm{JAC}}$ across time points, measured by two observers, error bars are SEM. B. Graph showing correlation of Inter-observer data for $A \cup C_{\text {power spectrum data. }}$ C. Bland-Altman plot showing the $95 \%$ limits of agreement in $A \cup C_{\text {power spectrum results. }}$ (Mean difference: thick solid line, mean \pm 2 standard deviations: dotted lines).

Figure 5. Graphs showing the repeated analysis of $A \cup C_{\text {power spectrum }}$ for the normal $(A, B, C)$ and high $(D, E, F) B M l$ subjects measured by the two observers.

Figure 6: Graph illustrating the difference in small bowel motility during fasting and immediately after feeding using the two different motility parameters across all the 15 subjects. A. The fasting and fed small bowel motility measured by $A \cup C_{\text {power spectrum. }} B$. The fasting and fed small bowel motility measured by $\mathrm{SD}_{\mathrm{JAC}}$. C. Correlation between the two parameters of the fasting and fed data. Individual subjects have the same colour coding across A and B. 


\section{List of abbreviations:}

$\begin{array}{ll}\text { AUC }_{\text {power spectrum }} & \text { Area under the power spectrum } \\ \text { ICC } & \text { Intra-class correlation coefficient } \\ \text { MRE } & \text { Magnetic resonance enterography } \\ \text { ROI } & \text { Region of interest } \\ \text { SD JAC } & \text { Standard deviation of the Jacobian }\end{array}$




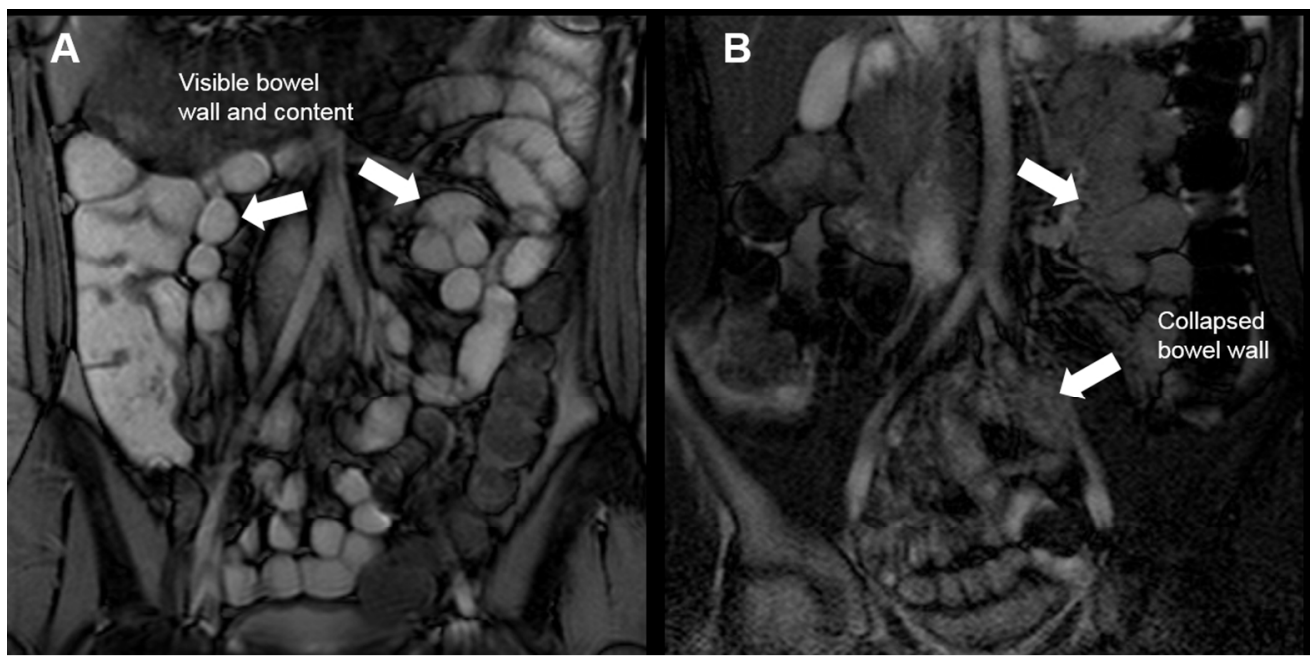

Figure 1. Figure illustrating the difference between $(A)$ the prepared bowel with clear definition of the bowel wall and bright luminal contents (data not from the current study, bowel preparation of $2 \%$ mannitol with $0.2 \%$ locust bean gum) and (B) the unprepared bowel with less visible bowel wall and brighter contents only when chyme moves into the segment. 


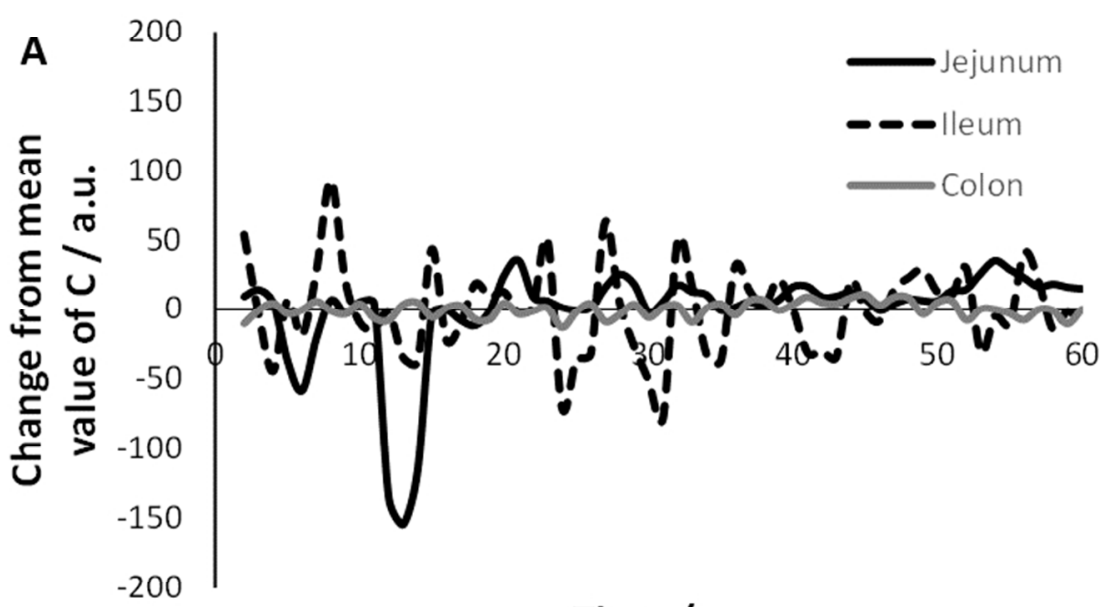

Time / s

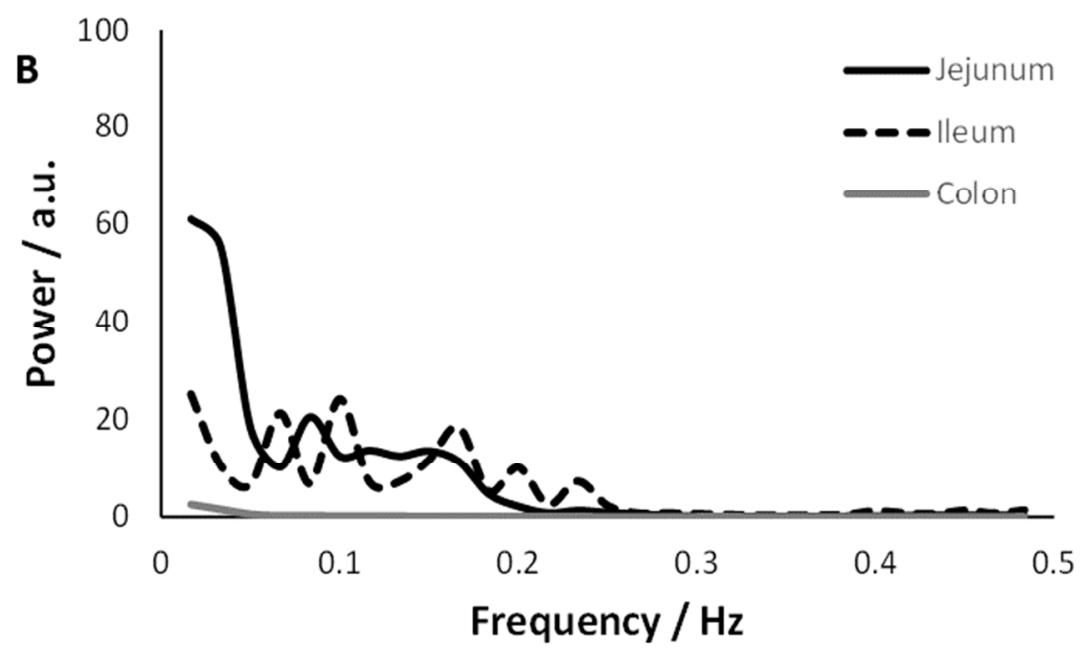

Figure $2 \mathrm{~A}$. Graph showing the variation in intensity of the $\mathrm{C}$ parameter with time for 3 abdominal regions. Solid black line is a small ROI from the upper small bowel (jejunum), dashed black line is a small ROI from the lower small bowel (ileum), solid grey line is a small ROI from the ascending colon representing a known low motility region of the GI tract. B. Corresponding power spectrum of the data in A. 
A: Fasting ( $\mathrm{AUC}_{\text {power spectrum) }}$
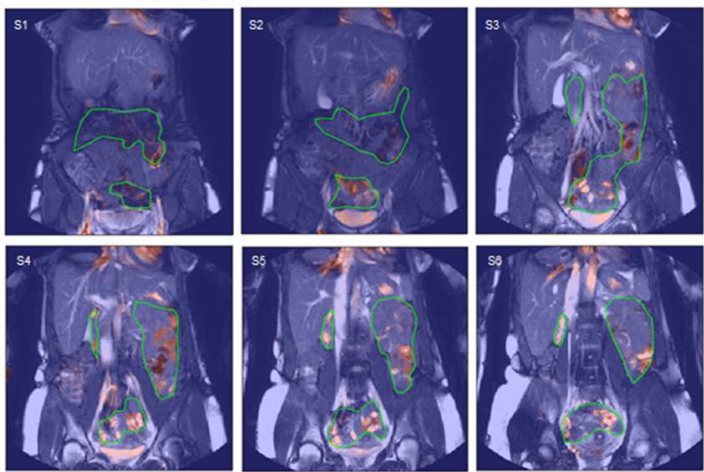

B: Fed (AUC $C_{\text {power spectrum })}$
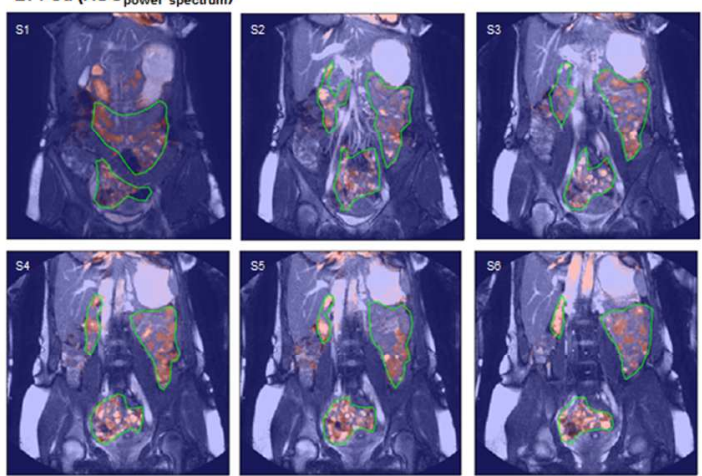

C: Fasting and fed state
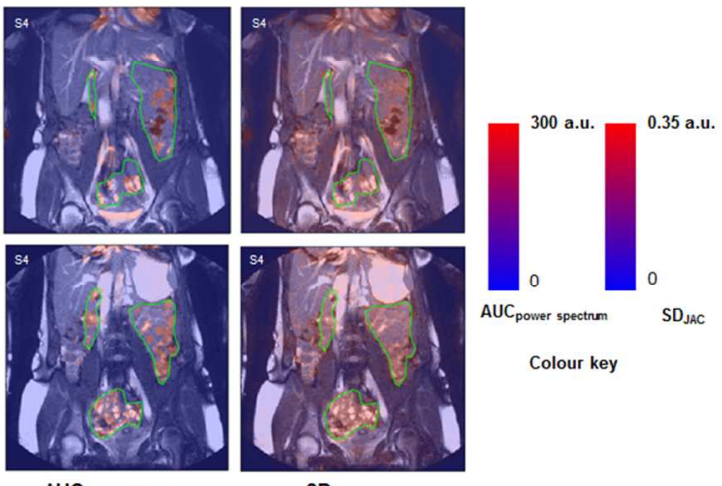

Figure 3. Example of motility maps generated by the software for a single volunteer across the 6 slices acquired, visualising the areas of high motility. A and B illustrate the fasting and the fed state motility maps.

$C$ represents the different motility maps generated by the AUCpower spectrum and SDJAC motility parameters. S: slice number. Regions of small bowel have been highlighted on the images. 

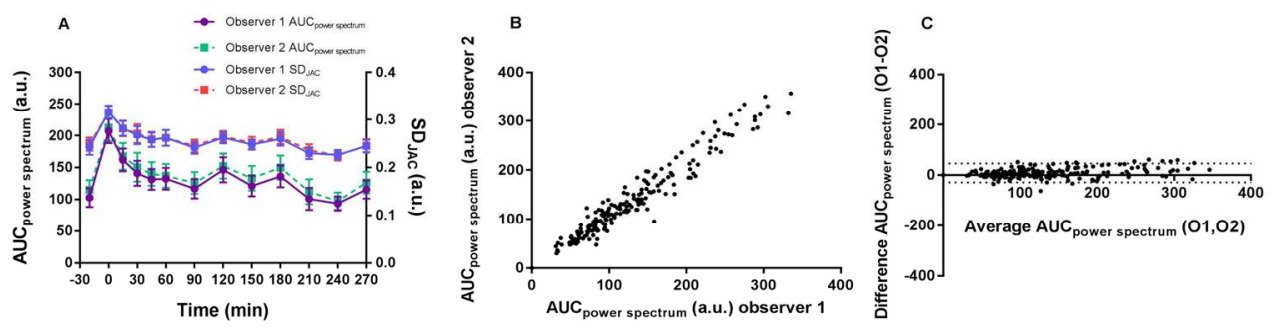

Figure 4. A. Graph showing the mean small bowel motility assessed with AUCpower spectrum and SDJAC across time points, measured by two observers, error bars are SEM. B. Graph showing correlation of Interobserver data for AUCpower spectrum data. C. Bland-Altman plot showing the $95 \%$ limits of agreement in AUCpower spectrum results. (Mean difference: thick solid line, mean \pm 2 standard deviations: dotted lines). 

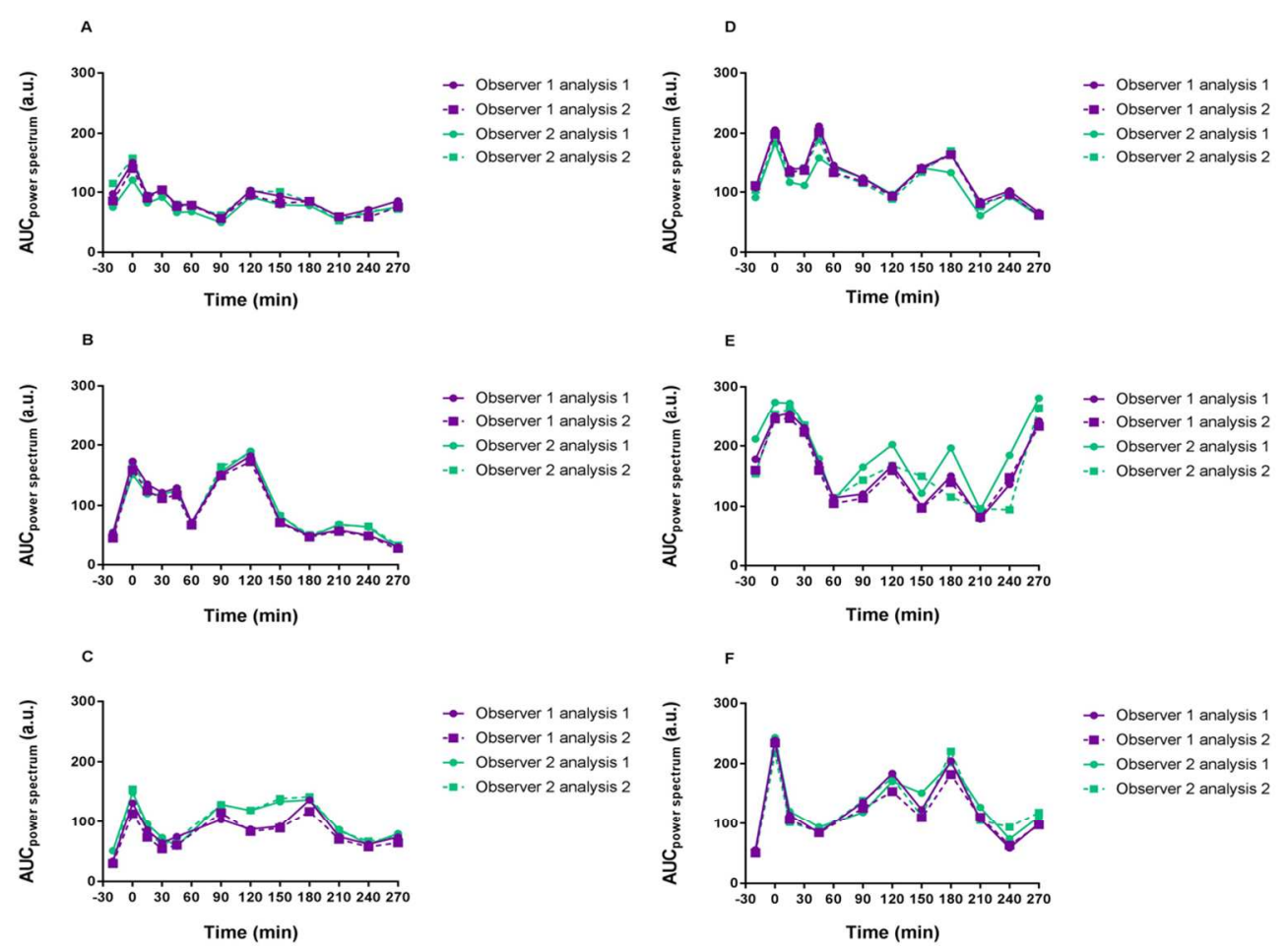

F

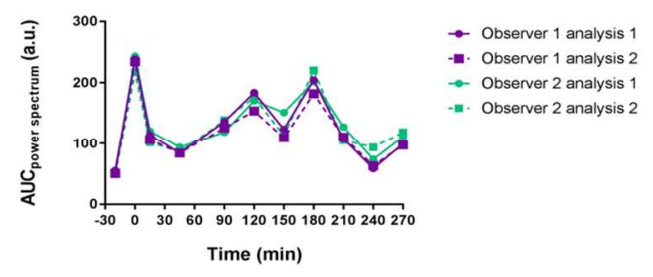

Figure 5. Graphs showing the repeated analysis of AUCpower spectrum for the normal $(A, B, C)$ and high $(D, E, F)$ BMI subjects measured by the two observers. 

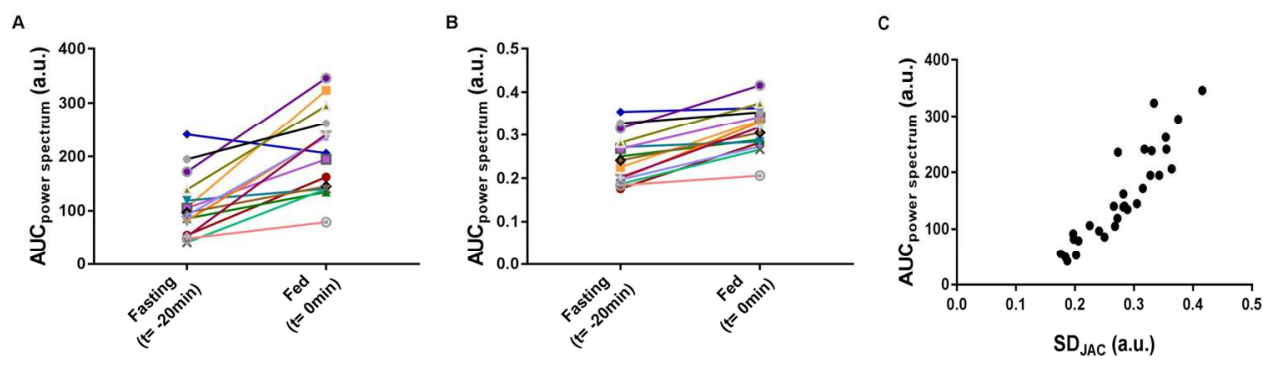

Figure 6: Graph illustrating the difference in small bowel motility during fasting and immediately after feeding using the two different motility parameters across all the 15 subjects. A. The fasting and fed small bowel motility measured by AUCpower spectrum. B. The fasting and fed small bowel motility measured by SDJAC. C. Correlation between the two parameters of the fasting and fed data. Individual subjects have the same colour coding across $\mathrm{A}$ and $\mathrm{B}$. 